\title{
Time to change weight gain recommendations for pregnant women with obesity
}

\author{
Sarah S. Comstock \\ Michigan State University, Department of Food Science \& Human Nutrition, East Lansing, Michigan, USA.
}

\begin{abstract}
Obesity during pregnancy is a major health problem in the United States. In this issue of the $J C l$, Most et al. fill an important gap in our understanding of energy homeostasis in pregnancy. The researchers measured energy intake, energy expenditure, and body composition in obese pregnant women. They demonstrated that energy intake need not increase in order for obese women to gain the recommended amounts of weight during pregnancy. Additionally, all of the gestational weight gain scenarios (inadequate, recommended, or excess) resulted in similar maternal and fetal perinatal outcomes. This evidence should guide new recommendations on this important topic.
\end{abstract}

remaining participants filling in all points in between. Only $25 \%$ of participants who gained within the current recommendation were in positive energy balance.

\section{Energy homeostasis in pregnancy and obesity}

During pregnancy, the human body grows a placenta and a fetus, and generates amniotic fluid, fat stores, and blood. Further, the uterus and mammary glands increase in size. Resting energy expenditure contributes to a majority (up to $70 \%$ ) of total daily energy expenditure and varies greatly from woman to woman $(8,9)$. As pregnancy progresses, the body begins to use fat for energy and becomes insulin resistant in order to provide glucose to the fetus (10). Often, additional energy intake and weight gain are required to support a pregnant woman and her developing infant. For instance, a lean woman without excess body fat will need additional energy intake to meet the high metabolic demand of pregnancy. However, in obese women the excess energy stored in adipose tissue can satisfy the metabolic demands of pregnancy. In some study populations, gestational weight gain is lower in women with high prepregnancy BMIs (11), whereas in others, excess gestational weight gain is common in women with obesity $(12,13)$.

Most et al. report that obese women do not need to ingest surplus calories in order to gain weight during pregnancy (7). In fact, the majority of participants in the study who gained within the 2009 IOM recommendation maintained a negative energy balance. Only women gaining in excess of the recommendation (36 of the 54 participants) accrued fat mass over the course of their pregnancy. The authors suggest that the conversion from fat mass to fat-free mass is efficient in pregnant women with obesity. This concept of metabolic efficiency during pregnancy is supported by prior research in normal weight and overweight pregnant women $(8,14)$, and implies that obese women who main-
Conflict of interest: SSC is named as an inventor on the following patents or patent applications: Mammalian milk osteopontin for enhancing immune responsiveness (patent number: 9956283; May 1, 2018); Modulation of immune function by dietary bovine lactoferrin (publication number: 20150246100; September 3, 2015); and Nutritional formulations using human milk oligosaccharides for modulating inflammation (publication number: 20160256479; September 8, 2016)

Copyright: () 2019, American Society for Clinical Investigation.

Reference information: / Clin Invest. 2019;129(11):4567-4569. https://doi.org/10.1172/JCI131932. 


\section{Table 1. Total weight gain recommendations for pregnant women with obesity}

$\begin{array}{lcc}\text { BMI category } & \text { Current IOM recommendation (Ibs) } & \text { Proposed recommendations (Ibs) } \\ \text { Obese, class 1 }(30-34.9) & 11-20 & 5-15 \\ \text { Obese, class 2 }(35-39.9) & 11-20 & <10 \\ \text { Obese, class 3 }(\geq 40) & 11-20 & \text { Maintain prepregnancy weight } \\ \text { In all cases, at a minimum, pregnant women should maintain their prepregnancy weight. }{ }^{A} \text { From } \\ \text { reference 1. }{ }^{B} \text { Based on references 6, 18. }\end{array}$

tain their body weight could have a healthy pregnancy with appropriate fetal development so long as specific nutrient requirements are met. Importantly, although Most et al. showed that energy balance affected adiposity, fetal growth remained unaffected (7). Despite these results, prospective energy intake intervention trials, powered to detect intervention effects on weight gain and perinatal outcomes, are warranted. Most and colleagues suggest that such interventions should result in an approximately $100 \mathrm{kcal} / \mathrm{d}$ energy deficit in order to effectively keep weight gain within the current recommendation (11-20 lbs) or an approximately 10\% energy deficit in order to achieve weight maintenance (7). Assessing the impact of fat localization such as on waist versus hip, and including obese women who have comorbidities such as hypertension, diabetes, and severe anemia, are additional avenues worth examining.

\section{Weight maintenance in obese pregnant women}

While maintaining weight may provide the lowest risk for obese pregnant women, losing weight could prove detrimental to perinatal outcomes. Two recent systematic reviews summarizing the results of 6 or 18 cohort studies addressed the issue of gestational weight gain for obese pregnant women $(15,16)$. In one, the authors conclude that gestational weight loss results in an increased likelihood of small for gestational age (SGA) infants as well as low birth weight (15). Conversely, the other showed that weight gain below the current IOM recommendation, without weight loss, may optimize perinatal outcomes by decreasing macrosomia as well as maternal morbidities such as gestational hypertension and preeclampsia (16). There is also evidence that, in those with moderate to high-risk obesity (class 2 or 3 ), inadequate weight gain does not increase SGA risk $(5,7,17)$. Indeed, a meta-analysis of data collected from cohorts in the U.S. and Europe found that the risk of adverse outcomes (preeclampsia, gestational hypertension, gestational diabetes, cesarean birth, preterm birth, and small or large for gestational age at birth) increased with BMI (regardless of BMI category) (18). Surprisingly, this risk was independent of gestational weight gain (18). A recent analysis of 12 million births using the United States Vital Statistics records compared outcomes from obese and ideal body weight pregnancies, and the results are striking (19). Negative maternal outcomes such as gestational hypertension and preeclampsia increase with gestational weight gain as well as obesity class. However, babies born to women with class 1 obesity who gained less than the recommended 11 pounds were slightly more likely to suffer from extremely low, very low, and low birth weight. Across the three obesity classes, the chance of infant macrosomia increased with gestational weight gain (19). Overall, this combined evidence suggests that the IOM recommendation for weight gain during pregnancy is too high for women with obesity, especially for those with class 2 or class 3 obesity (Table 1 ).

\section{Maternal and perinatal outcomes}

Despite indications that maintaining weight without gain in obese pregnant women results in healthy outcomes, the IOM has recommended potentially excessive weight gains (1). In this case, the desire to avoid negative perinatal outcomes for infants (preterm birth, low birth weight, and SGA at birth) may result in negative maternal outcomes (preeclampsia, gestational hypertension, gestational diabetes, cesarean birth). This tightrope walk is not a simple balancing act, as both less-gain and more-gain result in different reductions in negative perinatal outcomes (19). In the case of less gain, the pregnant woman benefits slightly more. In the case of more gain, the infant benefits slightly. Many analyses show that weight gains within the IOM recommendation protect from preterm birth, low birth weight, and SGA, prioritizing an infant-centric model $(6,16,18,19)$. On the other hand, these analyses also report that weight gains below the IOM recommendations protect the mother/infant dyad from large for gestational age, macrosomia, cesarean birth, preeclampsia, and gestational hypertension, prioritizing a dyad-centric model (16, 19). In the decade since the IOM recommendations were made, the medical field has taken a renewed interest in minimizing maternal morbidity. Recommending weight gains lower than current values may not only serve present-day standards but also the pregnant women themselves.

\section{Roles for nutrient intake or physical activity}

Most et al. reported no differences in physical activity, diet quality, or eating behavior by weight gain category (inadequate, recommended, excess) (7). However, the study was not powered to detect such differences. Prior secondary data analyses also demonstrate that complications such as low birth weight were more likely when pregnant women did not gain the IOM recommended weight, but in these studies dietary nutritional composition as well as physical activity levels were often unknown. It is possible that optimizing nutrient intake without weight gain could result in a healthy pregnancy and decreased risk for both the pregnant woman and her infant, thereby supporting a maternal-infant dyad-centric model. Future studies should carefully consider nutrient value beyond caloric intake as well as physical activity when evaluating perinatal outcomes within the context of weight gain.

\section{Conclusion}

Whether or not federal agencies or other organizations adjust weight gain recommendations for pregnant women with obesity, the fact remains that the majority of women with obesity gain in excess of the 2009 IOM recommendation $(19,20)$. 
A striking $67 \%$ of women in the study by Most et al. gained in excess of the IOM recommendation, which is similar to that reported elsewhere $(7,20)$. Thus, a continued challenge will be identifying successful strategies to prevent excessive weight gain in pregnant women with obesity.

Address correspondence to: Sarah S. Comstock, 469 Wilson Road, East Lansing, Michigan 48824, USA. Phone: 517.353.3415; Email: comsto37@msu.edu.

1. Rasmussen KM, Yaktine AL. Weight Gain During Pregnancy: Reexamining the Guidelines. Washington, DC, USA: National Academies Press; 2009.

2. Weight gain during pregnancy. Committee Opinion No. 548. American College of Obstetricians and Gynecologists. Obstet Gynecol. 2013; 121:210-212. http://www.acog.org/Resources_ And_Publications/Committee_Opinions_List.

3. Margerison Zilko CE, Rehkopf D, Abrams B. Association of maternal gestational weight gain with short- and long-term maternal and child health outcomes. Am JObstet Gynecol. 2010;202(6):574.e1-574.e8.

4. Oken E, Kleinman KP, Belfort MB, Hammitt JK, Gillman MW. Associations of gestational weight gain with short- and longer-term maternal and child health outcomes. Am JEpidemiol. 2009;170(2):173-180.

5. Bianco AT, Smilen SW, Davis Y, Lopez S, Lapinski R, Lockwood CJ. Pregnancy outcome and weight gain recommendations for the morbidly obese woman. Obstet Gynecol. 1998;91(1):97-102.

6. Robillard PY, Dekker G, Boukerrou M, Le Moullec N, Hulsey TC. Relationship between pre-pregnancy maternal BMI and optimal weight gain in singleton pregnancies. Heliyon. 2018;4(5):e00615.

7. Most J, et al. Evidence-based recommendations for energy intake in pregnant women with obesity. J Clin Invest. 2019;129(11):4682-4690.

8. Berggren EK, O'Tierney-Ginn P, Lewis S, Presley L, De-Mouzon SH, Catalano PM. Variations in resting energy expenditure: impact on gestational weight gain. Am JObstet Gynecol. 2017;217(4):445.e1-445.e6.

9. Most J, et al. Energy expenditure in pregnant women with obesity does not support energy intake recommendations. Obesity (Silver Spring). 2018;26(6):992-999.

10. Rees WD. Interactions between nutrients in the maternal diet and the implications for the long-term health of the offspring. Proc Nutr Soc. 2019;78(1):88-96.

11. Hutcheon JA, Platt RW, Abrams B, Himes KP, Simhan HN, Bodnar LM. Pregnancy weight gain charts for obese and overweight women. Obesity (Silver Spring). 2015;23(3):532-535.

12. Deputy NP, Sharma AJ, Kim SY. Gestational weight gain - United States, 2012 and 2013. MMWR Morb Mortal Wkly Rep. 2015;64(43):1215-1220.

13. Flegal KM, Kruszon-Moran D, Carroll MD, Fryar CD, Ogden CL. Trends in obesity among adults in the United States, 2005 to 2014. JAMA. 2016;315(21):2284-2291.
14. Abeysekera MV, Morris JA, Davis GK, O'Sullivan AJ. Alterations in energy homeostasis to favour adipose tissue gain: A longitudinal study in healthy pregnant women. Aust NZ J Obstet Gynaecol. 2016;56(1):42-48.

15. Kapadia MZ, Park CK, Beyene J, Giglia L, Maxwell C, McDonald SD. Weight loss instead of weight gain within the guidelines in obese women during pregnancy: a systematic review and meta-analyses of maternal and infant outcomes. PLoS ONE. 2015;10(7):e0132650.

16. Kapadia MZ, Park CK, Beyene J, Giglia L, Maxwell C, McDonald SD. Can we safely recommend gestational weight gain below the 2009 guidelines in obese women? A systematic review and meta-analysis. Obes Rev. 2015;16(3):189-206.

17. CDC. Defining adult overweight and obesity. http://www.cdc.gov/obesity/adult/defining.html. Updated April 11, 2017. Accessed August 30, 2019.

18. LifeCycle Project-Maternal Obesity Childhood Outcomes Study Group, et al. Association of gestational weight gain with adverse maternal and infant outcomes. JAMA. 2019;321(17):1702-1715.

19. Thompson AM, Thompson JA. An evaluation of whether a gestational weight gain of 5 to 9 $\mathrm{kg}$ for obese women optimizes maternal and neonatal health risks. BMC Pregnancy Childbirth. 2019;19(1):126.

20. Chuang CH, Stengel MR, Hwang SW, Velott D, Kjerulff KH, Kraschnewski JL. Behaviours of overweight and obese women during pregnancy who achieve and exceed recommended gestational weight gain. Obes Res Clin Pract. 2014;8(6):e577-e583. 